\title{
ASPP2 inhibits tumor growth by repressing the mevalonate pathway in hepatocellular carcinoma
}

\author{
Beibei Liang ${ }^{1}$, Rui Chen², Shaohua Song ${ }^{3}$, Hao Wang ${ }^{4}$, Guowei Sun ${ }^{5}$, Hao Yang ${ }^{1}$, Wei Jing ${ }^{6}$, Xuyu Zhou ${ }^{6}$, Zhiren Fu ${ }^{3}$,
} Gang Huang ${ }^{1}$ and Jian Zhao ${ }^{1}$

\begin{abstract}
Cancer is, fundamentally, a disorder of cell growth and proliferation, which requires adequate supplies of energy and nutrients. In this study, we report that the haplo-insufficient tumor suppressor ASPP2, a p53 activator, negatively regulates the mevalonate pathway to mediate its inhibitory effect on tumor growth in hepatocellular carcinoma (HCC). Gene expression profile analysis revealed that the expression of key enzymes in the mevalonate pathway were increased when ASPP2 was downregulated. HCC cells gained higher cholesterol levels and enhanced tumor-initiating capability in response to the depletion of ASPP2. Simvastatin, a mevalonate pathway inhibitor, efficiently abrogated ASPP2 depletion-induced anchorage-independent cell proliferation, resistance to chemotherapy drugs in vitro, and tumor growth in xenografted nude mice. Mechanistically, ASPP2 interacts with SREBP-2 in the nucleus and restricts the transcriptional activity of SREBP-2 on its target genes, which include key enzymes involved in the mevalonate pathway. Moreover, clinical data revealed better prognosis in patients with high levels of ASPP2 and low levels of the mevalonate pathway enzyme HMGCR. Our findings provide functional and mechanistic insights into the critical role of ASPP2 in the regulation of the mevalonate pathway and the importance of this pathway in tumor initiation and tumor growth, which may provide a new therapeutic opportunity for HCC.
\end{abstract}

\section{Introduction}

Dysregulation of a wide range of metabolic pathways can be involved in carcinogenesis ${ }^{1}$. For example, cholesterol has a critical role in maintaining the stability and architecture of the plasma membrane and is needed by highly proliferative cancer cells ${ }^{2}$. Tumor cell membranes have been found to be enriched in cholesterol, suggesting that enhanced cholesterol utilization is an important feature of malignant and perhaps metastatic tumors ${ }^{3}$. Mutational data from The Cancer Genome Atlas (TCGA) project also implicates cholesterol metabolism in cancer

\footnotetext{
Correspondence: Gang Huang (huangsumhs@163.com) or Jian Zhao (j-zhao@vip.126.com)

'Shanghai Key Laboratory of Molecular Imaging, Shanghai University of Medicine and Health Sciences, 201318 Shanghai, China

${ }^{2}$ Angecon Biotechnology Limited, 201318 Shanghai, China Full list of author information is available at the end of the article. These authors contributed equally: Beibei Liang, Rui Chen, Shaohua Song, Hao Wang

Edited by: A. Finazzi-Agrò
}

development ${ }^{4}$. The mevalonate pathway (MVA) is the essential metabolic pathway that converts acetyl-CoA to sterols such as cholesterol and non-sterol isoprenoids. Clinical and experimental findings suggest that malignant cells and tissues need higher amounts of cholesterol and intermediates of the mevalonate pathway than their normal counterparts ${ }^{5,6}$. Some types of cancers, such as hepatocellular carcinoma (HCC), depend on mevalonate pathway for growth. For example, inhibition of mevalonate metabolism suppresses tumor initiation and growth in a myc-transgenic murine $\mathrm{HCC}$ model ${ }^{7}$, activation of the mevalonate pathway also plays an essential role in liver tumorigenesis caused by p53 loss in mice ${ }^{8}$.

The mevalonate pathway is tightly regulated by a series of enzymes. 3-hydroxy-3-methylglutaryl-CoA reductase (HMGCR) is the rate-limiting enzyme, and can be blocked by statins ${ }^{9}$; indeed, such inhibition has antitumor effects in multiple tumor types ${ }^{9}$. Clinical observations have

\section{(c) The Author(s) 2019}

(c) (i) Open Access This article is licensed under a Creative Commons Attribution 4.0 International License, which permits use, sharing, adaptation, distribution and reproduction in any medium or format, as long as you give appropriate credit to the original author(s) and the source, provide a link to the Creative Commons license, and indicate if changes were made. The images or other third party material in this article are included in the article's Creative Commons license, unless indicated otherwise in a credit line to the material. If material is not included in the article's Creative Commons license and your intended use is not permitted by statutory regulation or exceeds the permitted use, you will need to obtain permission directly from the copyright holder. To view a copy of this license, visit http://creativecommons.org/licenses/by/4.0/. 
shown a negative association between the use of statins and the risk of developing liver cancer ${ }^{10}$.

Transcriptional activation of key rate limiting enzymes, like HMGCR, in mevalonate pathway is controlled by sterol-regulated cleavage of the membrane-bound transcription factor SREBP-2 (sterol regulated element binding protein-2) ${ }^{11-13}$. Depletion of intracellular sterols enhances SREBP-2 translocation from the endoplasmic reticulum (ER) to the Golgi apparatus, leading to subsequent cleavage to its active mature form and nuclear translocation ${ }^{14}$. SREBP-2 binds to sterol regulatory elements (SREs) in its target gene promoters to activate their transcription.

ASPP2 is a member of the ankyrin-repeat, SH3-domain, and proline-rich region-containing protein (ASPP) family, and is a haplo-insufficient tumor suppressor ${ }^{15,16}$. Aberrant expression of ASPP2 has been found in various human cancers ${ }^{17}$. Our previous study found that ASPP2 is downregulated by DNA methylation in $\mathrm{HCC}^{18}$. ASPP2 inhibits tumor growth and metastasis through regulation of apoptosis, autophagy, and epithelial plasticity ${ }^{16,19-21}$.
However, it remains unknown whether ASPP2 is involved in the regulation of mevalonate metabolism, which may contribute to its inhibitory effect on tumor progression. In this study, we provide evidence that downregulation of ASPP2 enhances tumor-initiating capabilities and tumor growth by promoting mevalonate metabolism in HCC.

\section{Results}

Downregulation of ASPP2 enhances mevalonate pathway gene expression and cholesterol biosynthesis in HCC cells

To explore the mechanisms driving the inhibitory effects of ASPP2 on tumor growth and progression, we performed a microarray analysis to identify the potential down-stream targets of ASPP2 by comparing gene expression in ASPP2-depleted HCC-LM3 cells and parent cells. Interestingly, GO analysis revealed a significant negative correlation between ASPP2 expression and terpenoid backbone biosynthesis and steroid biosynthesis (Fig. 1a). Expression of genes involved in mevalonate pathway, such as Hmgcs1, Hmgcr, Mvk, Mvd, and Idi1, appeared to be significantly enriched $(P<0.05$, FDR $<$

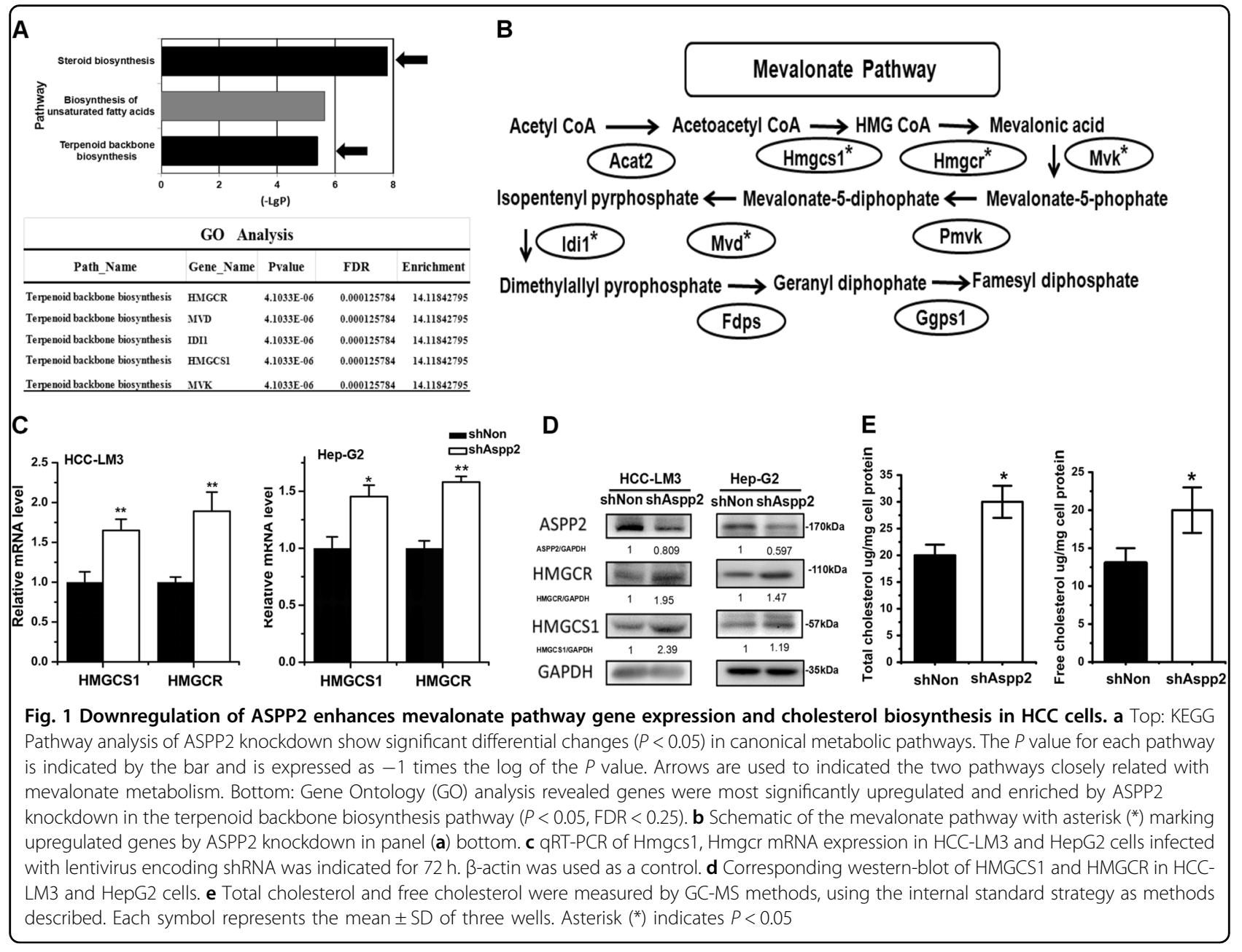




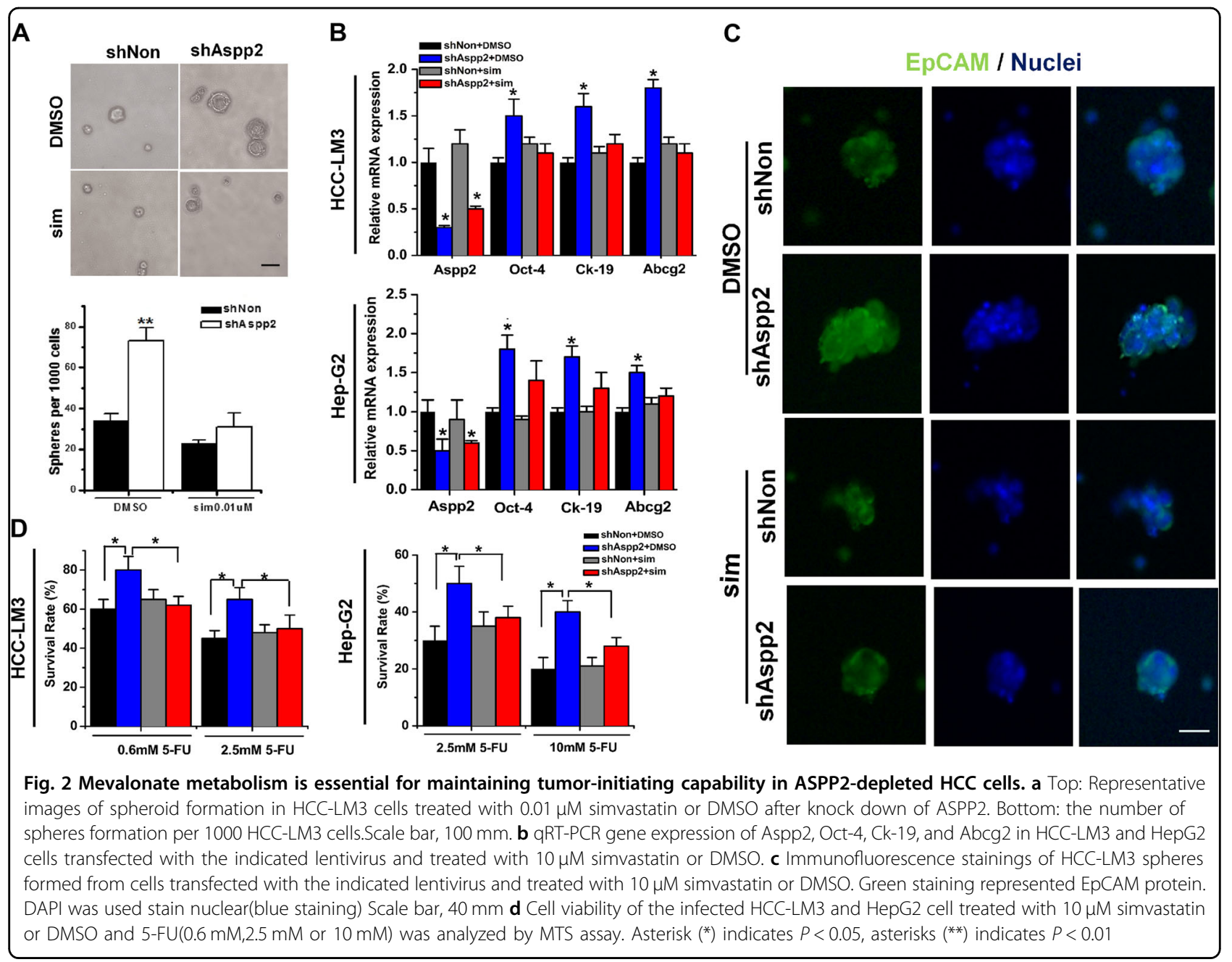

0.25) in ASPP2-depleted HCC-LM3 cells (Fig. 1b). To validate this finding, we examined the expression of HMG-CoA reductase (HMGCR) and HMG-CoA synthase1 (HMGCS1), which are the rate-limiting enzymes for mevalonate pathway ${ }^{22,23}$, by real-time PCR and Western Blotting. The results revealed that the mRNA and protein levels of HMGCR and HMGCS1 were notably elevated in ASPP2-depleted HCC-LM3 and HepG2 cells (Figs. 1c and d). Moreover, knockdown of ASPP2 led to a higher levels of free cholesterol and total cholesterol in HCC-LM3 cells by GC-MS analysis (Fig. 1e). Taken together, these observations suggest that downregulation of ASPP2 in HCC cells enhances mevalonate pathway and cholesterol biosynthesis.

\section{Mevalonate metabolism is essential for maintaining tumor-initiating capability in ASPP2-depleted HCC cells}

Mevalonate metabolism disorder has been linked to the development and tumor-initiating capability in various cancers $^{24-26}$. We have previously demonstrated that downregulation of ASPP2 conferred HCC cells with stem cell-like properties ${ }^{27}$. To verify the importance of mevalonate metabolism in ASPP2-depletion induced tumorinitiating capability in HCC cells, we inhibited mevalonate metabolism with simvastatin, a type of statin that can inhibit HMGCR to deplete precursors of the mevalonate pathway and lower cholesterol levels ${ }^{28,29}$. Treatment with simvastatin had no effect on cell proliferation in HCCLM3 grown in monolayer culture (Supplementary Fig. 1). However, in a suspension culture system, the numbers and the size of cell spheres were greatly decreased in simvastatin-treated ASPP2-depleted HCC-LM3 cells (Fig. 2a), suggesting decreased self-renewal. EpCAM is considered as a putative maker for tumor-initiating cells in $\mathrm{HCC}^{30}$, as EpCAM-positive $\mathrm{HCC}$ cells possess progenitor cell features ${ }^{31}$. Simvastatin supplementation abrogated ASPP2 depletion-induced EpCAM mRNA and protein expression in HCC-LM3 cells (Supplementary Fig. 2), as well as EpCAM expression in the surface of HCC-LM3 spheres (Fig. 2c). In addition, we assessed the expression of stemness-associated genes by qRT-PCR. ASPP2 depletion resulted in upregulation of transcription 

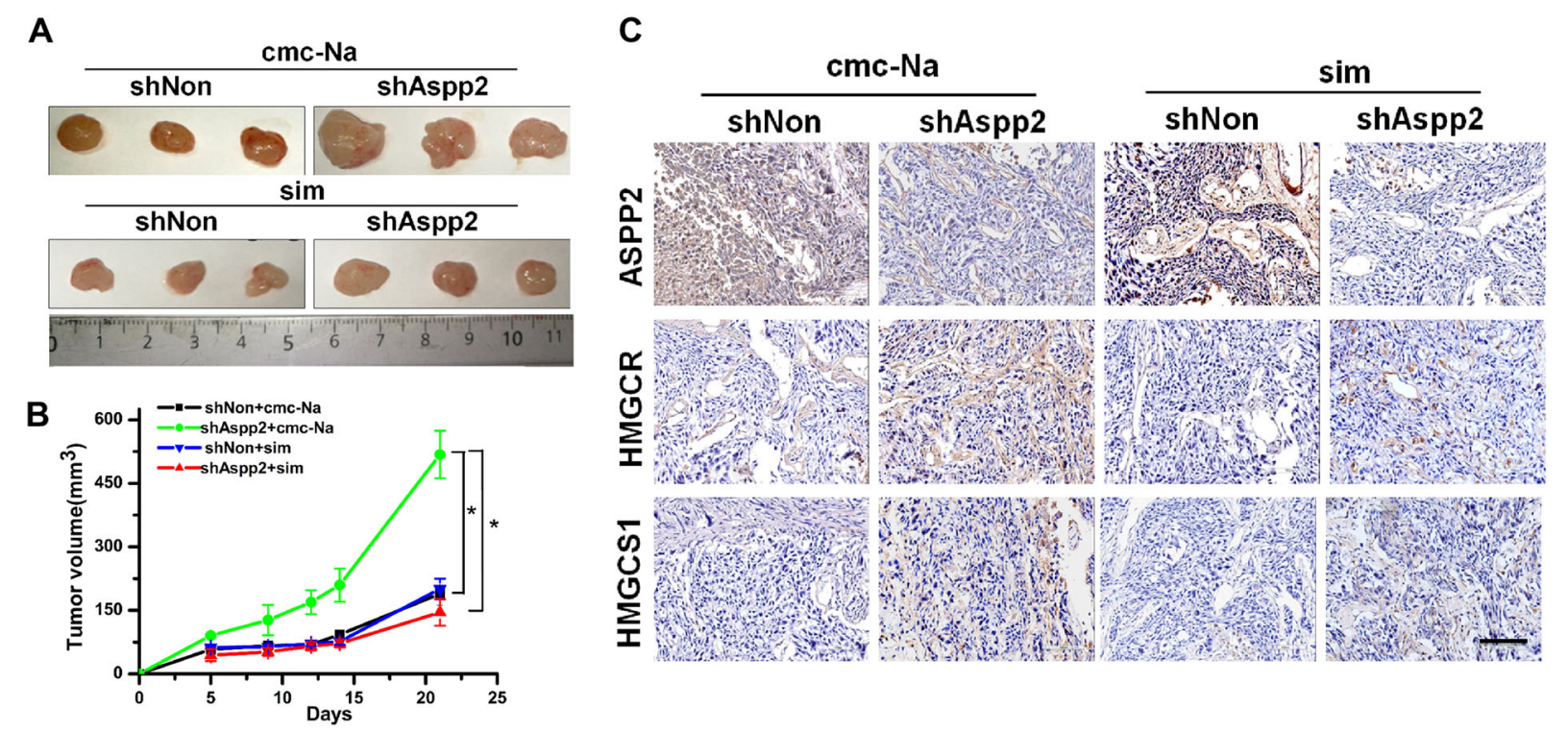

Fig. 3 Downregulation of ASPP2 promoted tumor growth in vivo by activating mevalonate biosynthesis. a Representative dissected tumors from nude mice treated without (top) or with (bottom) simvastatin for 3 weeks and corresponding volume measurement, $\mathbf{b}$ Asterisk (*) indicates $P<$ 0.05. c Immunohistochemical staining of tumors derived from nude mice for ASPP2, HMGCR, and HMGCS1 (magnification, X200). ASPP2-silenced groups exhibited increased HMGCR and HMGCS-1 expression. These alterations were compromised in ASPP2-silenced groups treated with simvastatin

factor Oct4, ATP-binding cassette transporter Abcg2, and Ck-19, a putative marker of tumor-initiating HCC cells, in HCC-LM3 and HepG2 cells. These alterations were compromised by simvastatin treatment (Fig. 2b). Resistance to chemotherapy has been regarded as another property of tumor initiating cells. Compared to control cells, ASPP2-depleted HCC-LM3 and HepG2 cells displayed significantly higher resistance to 5 -FU treatment. However, this resistance was decreased by simvastatin (Fig. 2d).

To further confirm that the mevalonate pathway contributes to tumor growth of ASPP2-depletion HCC cells in vivo, HCC-LM3 cells expressing shASPP2 or shNon were injected into the flank of nude mice, treated with or without simvastatin. Twenty one days after the xenograft, simvastatin significantly delayed tumor growth in ASPP2silenced groups (Fig. 3a, b). In addition, immunohistochemical staining showed tumors originated from ASPP2silenced groups exhibited increased HMGCR and HMGCS-1 expression (Fig. 3c). These data demonstrated that the mevalonate pathway is critical for maintaining tumor-initiating capability in ASPP2-depleted HCC cells.

\section{ASPP2 interacts with SREBP-2 to regulate mevalonate metabolism}

Microarray analysis revealed that several mevalonate pathway genes appeared to be transcriptionally upregulated in ASPP2 depleted HCC-LM3 cells (Fig. 1a), and we hypothesized that ASPP2 is an upstream regulator of mevalonate pathway, probably through regulation of SREBP-2. It has been demonstrated that SREBP-2 is a predominant regulator of the mevalonate pathway ${ }^{32}$. SREBP-2 is located entirely in the nucleus of HCC-LM3 cells, and ASPP2 could be detected in both the nucleus and cytoplasm in HCC-LM3 cells (Fig. 4a). Interestingly, we found SREBP-2 co-localized with endogenous ASPP2 in the nucleus of HCC-LM3 cells (Fig. 4a). Moreover, endogenous ASPP2 was found to interact with SREBP-2 in HCC-LM3 as detected by co-immunoprecipitation in total cell lysates or nuclear lysates (Fig. 4b). The separation of nuclear proteins was confirmed by the low expression of cytoplasmic protein $\alpha$-tubulin and the high expression of nuclear protein Lamin B1 (Supplementary Fig. 3). Depletion of ASPP2 did not reduce the protein level of SREBP-2, however the interaction between ASPP2 and SREBP-2 was greatly reduced (Fig. 4b). This interaction was further confirmed by ectopic expression of V5tagged ASPP2 and HA-tagged SREBP-2 in HEK293T cells (Fig. 4c, d). To explore whether ASPP2 regulates SREBP-2 transcriptional activity, we generated luciferase reporter constructs containing the hamster HMGCS1 promoter, the human LDLR promoter, or three sterol regulatory elements (3SRE) that bind SREBP- ${ }^{13}$. Ectopic expression of ASPP2 in HCC-LM3 and Huh-7 greatly reduced 3SRE, HMGCS1 and LDLR promoter activities, while depletion of ASPP2 had the reverse effect on their activities (Fig. 4d). ASPP2 depletion-induced HMGCR and HMGCS-1 expression were ablated when Srebp-2 was 


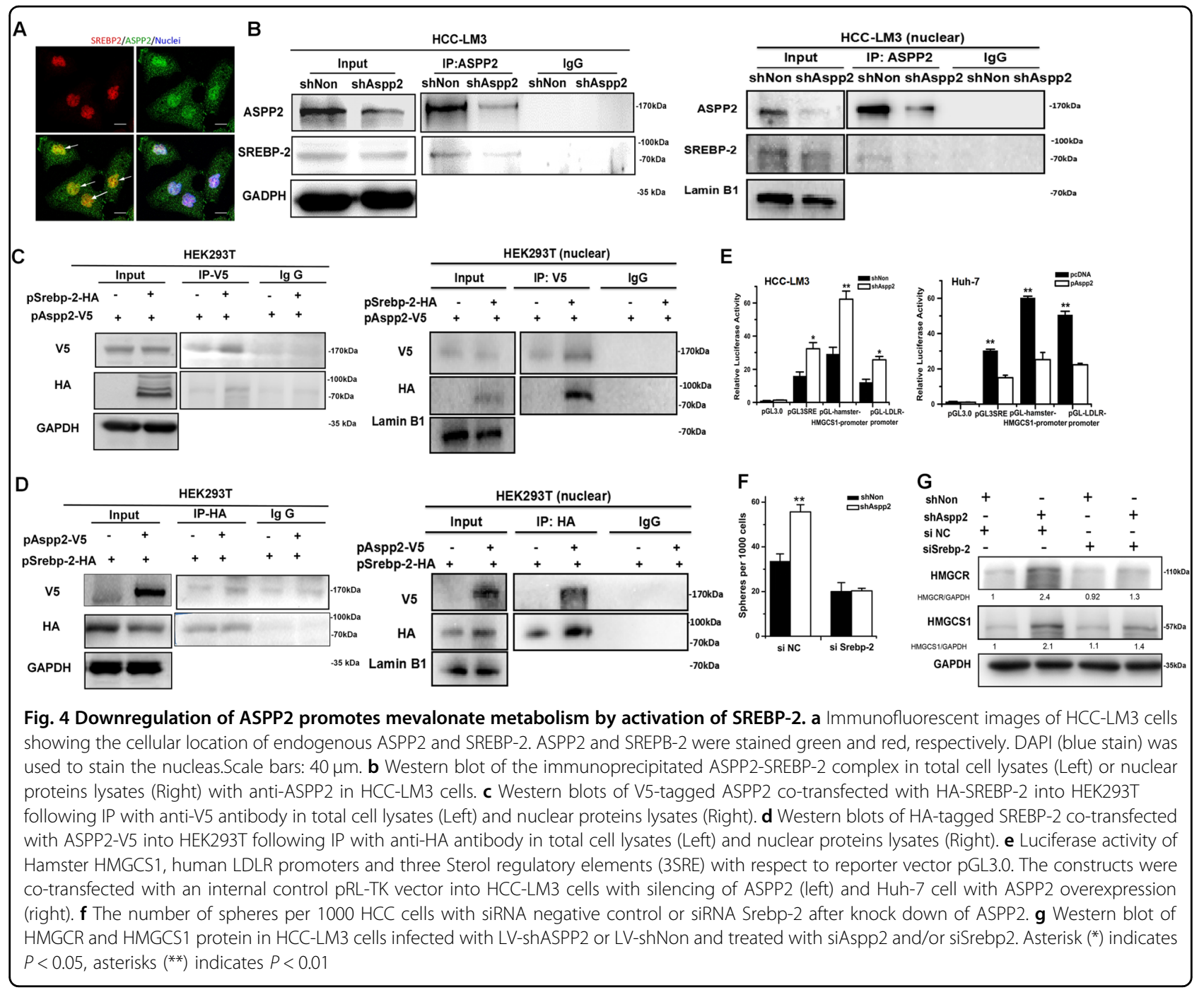

knocked down (Fig. 4f). Moreover, siRNA targeting Srebp2 abrogated ASPP2 depletion-induced sphere formation in HCC-LM3 cells (Fig. 4e). These results suggested that ASPP2 interacts with SREBP-2 and serves as a negative regulator of mevalonate metabolism.

\section{ASPP2-regulated mevalonate metabolism is associated with tumor progression and provides prognostic value}

To assess the clinical impact of ASPP2-regulated mevalonate metabolism, we examined ASPP2 and HMGCR protein expression in $80 \mathrm{HCC}$ tissues by immunohistochemistry. The clinical pathological parameters of all HCC patients are shown in Supplementary Table 1 . In 30 cases, the tumor samples displayed high ASPP2 expression. Most of the ASPP2 was located in the cytoplasm (Fig. 5a), but 17 cases had strong nuclear staining of ASPP2 (Fig. 5c). Thirty-eight percent (30/80) cases showed low ASPP2 expression and high expression of HMGCR, while 23\% (18/80) cases showed high ASPP2 expression and low HMGCR expression. Thus, the expression of ASPP2 and HMGCR is inversely correlated $(P<0.05$; Fig. $5 b)$. Among the cases with ASPP2 in the nucleus, $70.6 \%(12 / 17)$ had low HMGCR expression, in contrast to only $46.2 \%(6 / 13)$ of cases with cytoplasmic ASPP2 (Fig. 5d). This suggests that nuclear ASPP2 may be more likely to negatively regulate HMGCR expression.

The relationships between ASPP2 and HMGCR expression and clinical features are statistically analyzed in Table 1. A significant correlation between HMGCR expression and tumor volume was observed $(P=0.003)$ : patients with high HMGCR expression were prone to have a larger tumor volume. Further, patients with ASPP2-high and HMGCR-low expression had smaller tumor volumes $(P=0.008)$ and less cirrhosis $(P=0.018)$. Consistent with these findings, patients with ASPP2-high and HMGCR-low expression exhibited the best recurrence-free survival (RFS), as well as overall survival (OS) (Fig. 5e). These data suggest that ASPP2-regulated 


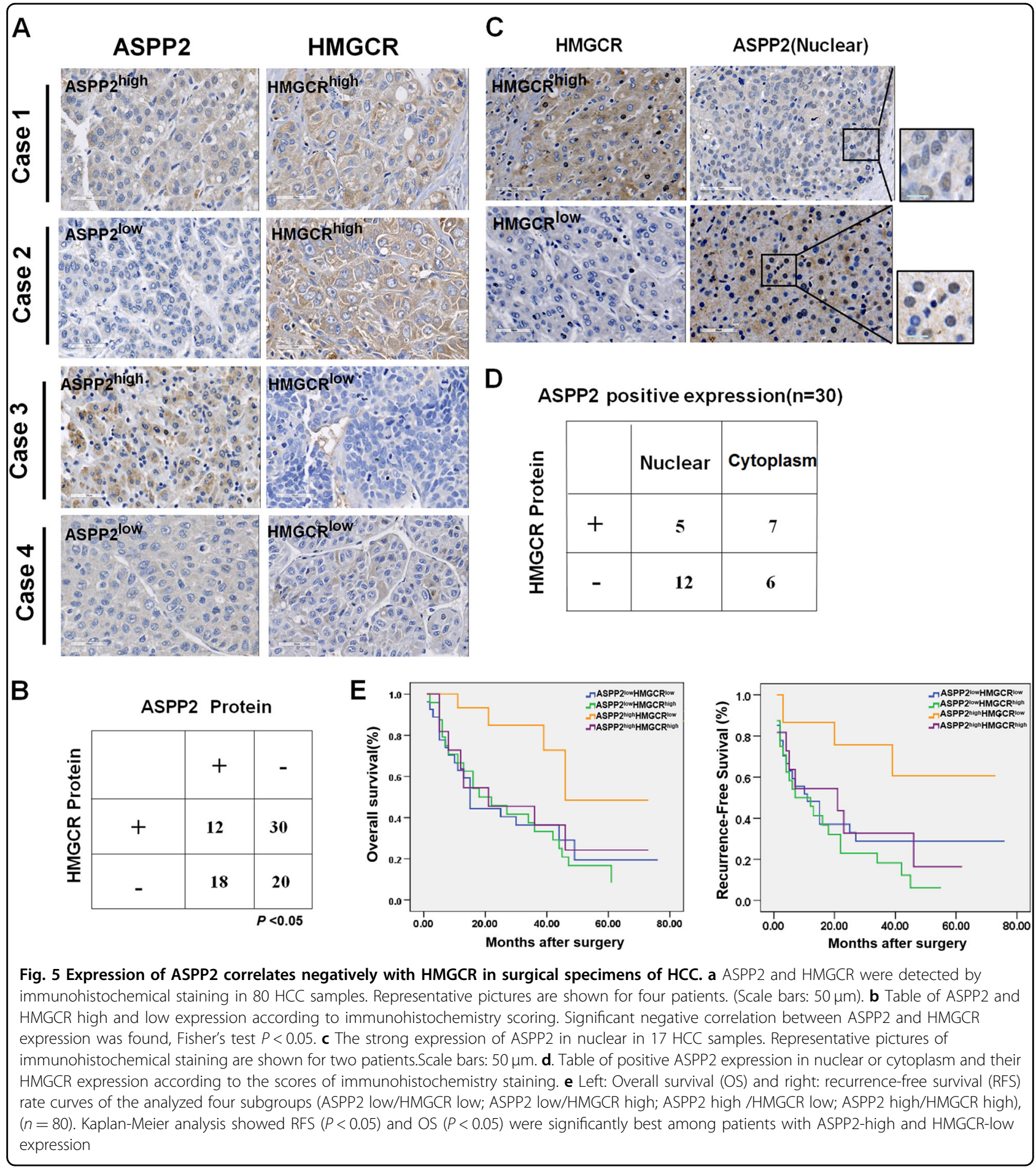

mevalonate metabolism contributes to tumor progression in HCC.

In univariate analysis, tumor size, AJCC stage, ASPP2, and HMGCR expression status are to be prognostic factors for RFS and OS $(P<0.05$, Table 2$)$. In multivariate analysis, tumor size, ASPP2, and HMGCR expression status were shown as significant independent predictors of RFS and OS (Table 3). Patients with HMGCR-high expression were 2.530 times more likely to suffer from relapse than patients with HMGCR-low expression (hazard ratio, 2.530; 95\% confidence interval, 1.355-4.725). Patients with ASPP2-high expression were about 0.453 times less at risk of relapse than patients with ASPP2-low expression (hazard ratio, 0.453; 95\% 
Table 1 The associations of ASPP2 and HMGCR expression with clinicopathologic characteristics in 80 patients with HCC

\begin{tabular}{|c|c|c|c|c|c|c|c|c|c|}
\hline & \multicolumn{3}{|c|}{ Whole study $(n=80)$} & \multicolumn{3}{|c|}{ ASPP2 negative group $(n=50)$} & \multicolumn{3}{|c|}{ ASPP2 positive group $(n=30)$} \\
\hline & \multicolumn{2}{|c|}{ HMGCR expression } & \multirow[t]{2}{*}{$P$} & \multicolumn{2}{|c|}{ HMGCR expression } & \multirow[t]{2}{*}{$P$} & \multicolumn{2}{|c|}{ HMGCR expression } & \multirow[t]{2}{*}{$P$} \\
\hline & $\begin{array}{l}\text { Negative } \\
(n=38)\end{array}$ & $\begin{array}{l}\text { Positive } \\
(n=42)\end{array}$ & & $\begin{array}{l}\text { Negative } \\
(n=20)\end{array}$ & $\begin{array}{l}\text { Positive } \\
(n=30)\end{array}$ & & $\begin{array}{l}\text { Negative } \\
(n=18)\end{array}$ & $\begin{array}{l}\text { Positive } \\
(n=12)\end{array}$ & \\
\hline \multicolumn{10}{|l|}{ Sex } \\
\hline Male & 30 & 29 & 0.315 & 14 & 19 & 0.625 & 16 & 10 & 1.0 \\
\hline Female & 8 & 13 & & 6 & 11 & & 2 & 2 & \\
\hline \multicolumn{10}{|l|}{ Age (years) } \\
\hline$<50$ & 11 & 21 & 0.055 & 4 & 17 & 0.023 & 7 & 4 & 1.0 \\
\hline$\geq 50$ & 27 & 21 & & 16 & 13 & & 11 & 8 & \\
\hline \multicolumn{10}{|l|}{$\mathrm{HBsAg}$} \\
\hline Negative & 6 & 8 & 0.702 & 3 & 7 & 0.718 & 3 & 1 & 0.632 \\
\hline Positive & 32 & 34 & & 17 & 23 & & 15 & 11 & \\
\hline \multicolumn{10}{|l|}{ AFP (ng/ml) } \\
\hline$\leq 400$ & 23 & 18 & 0.114 & 12 & 13 & 0.248 & 11 & 5 & 0.457 \\
\hline$>400$ & 15 & 24 & & 8 & 17 & & 7 & 7 & \\
\hline \multicolumn{10}{|l|}{ Tumor volume $\left(\mathrm{cm}^{3}\right)$} \\
\hline$\leq 5$ & 28 & 17 & 0.003 & 14 & 14 & 0.103 & 14 & 3 & 0.008 \\
\hline$>5$ & 10 & 25 & & 6 & 16 & & 4 & 9 & \\
\hline \multicolumn{10}{|l|}{ Cirrhosis } \\
\hline- & 13 & 10 & 0.305 & 3 & 9 & 0.224 & 10 & 1 & 0.018 \\
\hline+ & 25 & 32 & & 17 & 21 & & 8 & 11 & \\
\hline \multicolumn{10}{|l|}{ AJCC stage } \\
\hline Stage I-II & 15 & 19 & 0.602 & 7 & 15 & 0.295 & 8 & 4 & 0.709 \\
\hline Stage III-IV & 23 & 23 & & 13 & 15 & & 10 & 8 & \\
\hline Recurrence time (months) & & & & 85 & & & & & \\
\hline$\leq 6$ & 11 & 17 & 0.28 & 7 & 13 & 0.556 & 4 & 4 & 0.678 \\
\hline$>6$ & 27 & 25 & & 13 & 17 & & 14 & 8 & \\
\hline
\end{tabular}

Note: $P$ values are two-tailed and based on the Pearson $x^{2}$ test

confidence interval, 0.235-0.873). Therefore, increased expression of HMGCR with decreased ASPP2 may serve as a prognostic indicator for patients with HCC.

\section{Discussion}

Here we demonstrate that ASPP2, an activator of p53, regulates tumor-initiating capability and tumor growth by inhibiting SREBP-2-mediated mevalonate metabolism in HCC. HCC cells gained higher cholesterol levels, increased cancer stemness characters and tumor growth by lentivirus-mediated downregulation of ASPP2. Simvastatin, a mevalonate metabolism pathway inhibitor reversed the increased tumor growth and stemness characteristics of ASPP2-depleted cells.

$\mathrm{p} 53$, the gatekeeper for cell growth and division, acts via multiple pathways, such as cell-cycle arrest, cell death and senescence. Increasing evidence demonstrates that regulation of cellular metabolic pathways by p53 contributes to its tumor suppressor effects ${ }^{33}$. Notably, recent evidence shows that $\mathrm{p} 53$ represses mevalonate metabolism via inhibiting maturation of SREBP-2, which plays a crucial role in p53-mediated tumor suppression in a mouse model of liver tumorigenesis ${ }^{8}$. Moreover, p53 harboring cancer-derived missense mutations interacts with SREBP- 
Table 2 Univariate analyses of factors associated with recurrence-free survival and overall survival

\begin{tabular}{|c|c|c|c|c|}
\hline \multirow[t]{2}{*}{ Variables } & \multicolumn{2}{|l|}{ RFS } & \multicolumn{2}{|l|}{ OS } \\
\hline & Hazard ratio $(95 \% \mathrm{Cl})$ & $P$ & Hazard ratio $(95 \% \mathrm{Cl})$ & $P$ \\
\hline Gender (male vs. female) & $0.670(0.351-1.278)$ & 0.224 & $0.67(0.348-1.275)$ & 0.22 \\
\hline Age, years ( $\geq 50$ vs. $<50$ ) & $0.630(0.364-1.089)$ & 0.098 & $0.776(0.481-1.203)$ & 0.363 \\
\hline HbsAg (positive vs. negative) & $1.181(0.425-3.285)$ & 0.75 & $1.078(0.385-3.013)$ & 0.887 \\
\hline AFP, ng/ml (>400 vs. $\leq 400)$ & $1.713(0.988-2.970)$ & 0.055 & $1.519(0.876-2.634)$ & 0.136 \\
\hline Cirrhosis (yes vs. no) & $1.589(0.871-2.899)$ & 0.131 & $1.436(0.724-1.894)$ & 0.239 \\
\hline Tumor size, cm ( $\geq 5$ vs. $<5)$ & $3.124(1.692-5.768)$ & 0.00 & $2.79(1.523-5.110)$ & 0.001 \\
\hline AJCC stage (III-IV vs. I-II) & $2.080(1.108-3.903)$ & 0.023 & $1.981(1.056-3.717)$ & 0.033 \\
\hline ASPP2 (high vs. low) & $0.423(0.221-0.809)$ & 0.009 & $0.437(0.229-0.833)$ & 0.012 \\
\hline HMGCR (high vs. low) & $2.095(1.193-3.679)$ & 0.01 & $1.979(1.130-3.466)$ & 0.017 \\
\hline
\end{tabular}

Table 3 Multivariate analyses of factors associated with recurrence-free survival and overall survival

\begin{tabular}{lll}
\hline & Hazard ratio (95\% Cl) & $P$ \\
\hline RFS & & \\
Tumor size, cm ( $\geq 5$ vs. $<5)$ & $2.530(1.355-4.725)$ & 0.004 \\
ASPP2 (high vs. low) & $0.505(0.257-0.989)$ & 0.026 \\
HMGCR (high vs. low) & $2.182(1.147-4.150)$ & 0.017 \\
OS & & \\
Tumor size, cm ( $\geq 5$ vs. $<5)$ & $2.103(1.122-3.942)$ & 0.02 \\
ASPP2 (high vs. low) & $0.453(0.235-0.873)$ & 0.017 \\
HMGCR (high vs. low) & $2.530(1.355-4.725)$ & 0.004 \\
\hline
\end{tabular}

Multivariate analysis, cox proportional hazards regression model

Variables were adopted for their prognostic significance by univariate analysis and no obvious correlation between each other

2 to activate the mevalonate pathway, and this is implicated in maintaining a malignant phenotype in breast cancer ${ }^{34}$.

ASPP2 was first identified as an activator of the p53 family that regulates apoptosis ${ }^{35}$. Subsequently, ASPP2 was found to regulate a wide range of biological events by cooperating with specific partners, such as p73, Bcl-2, NF$k B$, Yes-associated protein-1, RAS, Par3, $\beta$-catenin, and Beclin-1. At cell membranes, through its $\mathrm{N}$-terminus ASPP2 binds Par3 to maintain the integrity of cell polarity $^{36,37}$, and binds the $\beta$-catenin-E-cadherin complex to regulate epithelial plasticity ${ }^{20}$. In the cytoplasm, ASPP2 binds RAS to regulate RAS signaling ${ }^{38,39}$, and interacts with ATG5 and Beclin 1 to regulate autophagy ${ }^{19,21}$. In the nucleus, ASPP2 binds p53 and p73 to stimulate apoptosis by its $\mathrm{C}$ terminus ${ }^{40}$. Phosphorylation of ASPP2 by the RAS/Raf/MAPK pathway causes the translocation of
ASPP2 from the cell membrane to cytosol/nucleus ${ }^{41}$. LPS stimulates ASPP2 translocation from the cytoplasm to nucleus in murine macrophages, and causes nuclear accumulation of ASPP2 at the blood-cerebral spinal fluid barrier in an LPS-induced maternal inflammation mouse model $^{42}$. The C terminus of the ASPP2 contains ankyrin repeats, which may facilitate ASPP2 to enter the nucleus through the RanGDP/Ankyrin Repeats binding nuclear import pathway ${ }^{43}$.

Here, we showed evidence that ASPP2 interacts with SREBP-2 in the nucleus and inhibits the transcriptional activation of mevalonate pathway genes by SREBP-2. HCC tissues with nuclear ASPP2 staining were more prone to have low HMGCR expression than HCC tissue with cytoplasmic ASPP2 staining, further supporting a negative effect of nuclear ASPP2 on the mevalonate pathway.

Previous observations in cancer cells have shown ASPP2 expression predominantly in the cytoplasm or at the cell membrane ${ }^{20}$. However, our datas showed that ASPP2 expression was both nuclear and cytoplasm in HCC-LM3 cells and in about $21 \%(17 / 80)$ of cases of HCC. Colocalization of ASPP2 and SREBP-2 in HCC-LM3 nucleus was observed. Previous studies have shown that the transcriptional activity of SREBP-2 could be regulated by interaction with mutant p53, Myc, and RB ${ }^{9}$. Two gain-offunction p53 mutations are able to interact with nuclear SREBP-2 and increase SREBP-2-mediated transcription of the mevalonate pathway genes ${ }^{34}$. Data from the Encyclopedia of DNA Elements (ENCODE) project has shown that MYC binds to promoters of mevalonate pathway genes in close proximity to SREBP-2, which may contribute to Myc-induced hepatocarcinogenesis. RB prevents the association of SREBP-2 with target gene promoter and serves as a negative regulator of mevalonate metabolism $^{44}$. Our finds suggest that ASPP2 is a novel 
negative regulator of SREBP-2. Given that ASPP2 interacts with p53 in the nucleus, the interaction between of ASPP2 and SREBP2 is p53 dependent or not still need further investigation.

Since the liver is responsible for about $80 \%$ of de novo cholesterol biosynthesis in humans, it is not surprising to see a critical role of the mevalonate pathway in the development of liver cancer. Our findings have identified a crucial role of ASPP2 in regulating mevalonate metabolism and its importance in tumor-initiating capability and tumor growth, which may provide novel therapeutic opportunities for HCC.

\section{Materials and methods Cell culture}

We purchased HEK293T cells from American Type Culture Collection (ATCC). HCC-LM3 (p53 wt), HepG2 (p53 wt), and Huh-7 (p53 mut) were obtained from the Liver Cancer Institute, Zhong Shan Hospital, Fudan University (Shanghai, People's Republic of China). All cells line had been identified and tested the mycoplasma contamination. These cells were maintained in DMEM (Gibco, Waltham, MA, USA) supplemented with 10\% (vol/ vol) FBS (Gibco) and kept at $37^{\circ} \mathrm{C}$ and $5 \% \mathrm{CO} 2$. Simvastatin was used to inhibit HMGCR to deplete precursors of the mevalonate pathway and lower cholesterol levels.

\section{Lentivirus shRNA and small interfering RNA construction}

We designed three pairs of cDNA oligonucleotides targeting ASPP2 mRNA expression, using web-based software from Invitrogen and InvivoGen Inc. After synthesis, we inserted these double-strand oligos into the vector $\mathrm{pENTR/U6} \mathrm{(Invitrogen)} \mathrm{and} \mathrm{sequenced} \mathrm{the}$ resulting plasmids to ensure the shRNA construct targeted human ASPP2 expression or were scrambled, which were generated and designated as LV-shASPP2 and LVshNon. Then, the plasmids were transfected into HCCLM3 cells and gene silencing efficiency was validated $48 \mathrm{~h}$ after transfection by real-time PCR and Western blot. Details about lentivirial vector production and lentivirus infection can be found in the supplementary material.

\section{Cholesterol analysis}

HCC-LM3 cells in 6-well plates were infected with LVshNon and LV-shAspp2. After three days, two group cells were collected. Free cholesterol and total cholesterol were measured by GC-MS methods, using the internal standard strategy. Each symbol represents the mean \pm SD of three wells. The experiments have been repeated at least three times.

\section{Luciferase reporter plasmids construction and luciferase reporter assays}

The luciferase reporter plasmids, pGL-hamsterHMGCS1-promoter and pGL-LDLR-promoter were designed and generated (details can be found in the supplementary material). The pGL3.0-enhancer (Promega) and the constructed luciferase reporter plasmids were transfected into HCC $\left(3 \times 10^{4}\right)$ cells in 48-well plates with the pRL-TK in triplicate by X-tremeGENE HP DNA Transfection Reagent. Then the cells were collected after $24 \mathrm{~h}$ transfection to measure the luciferase activities with the Dual Luciferase Reporter Assay System (Promega). All data are presented as the means \pm S.D. The experiments have been repeated at least three times.

\section{Tumor xenograft model}

After infection with LV-shNon and LV-shAspp2 (at a MOI of 50), HCC-LM3 cells $\left(5 \times 10^{6}\right)$ were implanted into the flank of nude mice by subcutaneous injection (male $\mathrm{BALB} / \mathrm{c} \mathrm{nu} / \mathrm{nu}$, six in each group). Tumors were wellestablished after 7 days. Simvastatin $(40 \mathrm{mg} / \mathrm{kg}$ body weight) in $0.5 \%$ CMC-Na (sodium carboxymethyl cellulose) was given to mice daily by intraperitoneal injection for 3 weeks. Then the mice were sacrificed and the tumor tissues were isolated for histopathology experiments.

\section{Sphere formation assay}

$0.4 \%$ bovine serum albumin, $4 \mu \mathrm{g} / \mathrm{mL}$ insulin (SigmaAldrich, St Louis, MO, USA), $20 \mathrm{ng} / \mathrm{mL}$ basic fibroblast growth factor (PeproTech), B27 (1:50; Invitrogen), and $20 \mathrm{ng} / \mathrm{mL}$ epidermal growth factor (PeproTech, Rocky Hill, NJ, USA) were supplemented to DMEM/F12 medium(Invitrogen, Carlsbad, CA, USA). Then we suspended cells infected with LV-shNon and LV-shAspp2 at a concentration of 5000 cells $/ \mathrm{mL}$ with DMEM/F12 medium. $0.01 \mu \mathrm{M}$ Simvastatin or DMSO was added to the medium. Five thousand cells were plated onto ultralow attachment plates (Corning, Corning, NY, USA) in different conditions. After one-week of incubation, sphere numbers were counted using an Olympus IX70 microscope.

\section{Immunofluorescence}

Cultured cells were washed 3 times with cold phosphate buffer saline (PBS), fixed with $4 \%$ paraformaldehyde, blocked and then incubated with ASPP2 antibody (1:200, A4480, Sigma-Aldrich, antigenic epitope was located at amino acids 691-1128 of ASPP2 of human species) and SREBP-2 antibody (1:150, N-19 sc8151, Santa Cruz, antigenic epitope was located in N-terminus of SREBP-2 of human species) overnight at $4{ }^{\circ} \mathrm{C}$. Corresponding Alexa 555-conjugated and/or Alexa 488-conjugated secondary antibodies (Invitrogen) were used to incubate the cells for $1 \mathrm{~h}$ at room temperature, then 4,6-diamidino-2-phenylindole (DAPI; Vector Laboratories Inc., Burlingame, CA) for $5 \mathrm{~min}$. Representative images were captured with LSM710 laser confocal microscope (Fudan University). Immunofluorescence on HCC-LM3 spheres was performed with EpCAM antibody (1:100, \#36746, Cell signal 
technology) using the same method described above, under an Olympus IX70 microscope. Details about Immunofluorescence detection of EpCAM can be found in the supplementary material and method.

\section{Immunoprecipitation assay}

After different treatment, cells were incubated with lysis buffer ( $50 \mathrm{mM}$ Tris $\mathrm{HCl}, 150 \mathrm{mM} \mathrm{NaCl}, 0.1 \%$ SDS, $1 \% \mathrm{NP}$ 40, $0.5 \%$ sodium deoxycholate) plus protease inhibitors $1 \mathrm{mM}$ PMSF (Sigma, P7626) and protease inhibitor cocktail tablets (Roche, 04693116001). Cells were lysed for $30 \mathrm{~min}$ on ice and centrifuged at $16,000 \times g$ for $20 \mathrm{~min}$ at $4{ }^{\circ} \mathrm{C}$. The cell lysates were incubated with antibody overnight and the immune complexes precipitated with protein $\mathrm{A} / \mathrm{G}$ agarose (Santa Cruz, sc-2003) for $3 \mathrm{~h}$ at $4{ }^{\circ} \mathrm{C}$. Complexes were washed in lysis buffer $(5 \times 5 \mathrm{~min})$. Immunoprecipitated proteins were analyzed by Western blotting.

\section{Immunoprecipitation of nuclear proteins}

The nuclear proteins of treated HCC-LM3 or HEK293T cells were isolated by the Nuclear Complex CoIP Kit (Active Motif, 54001) according to the manufacturer's instructions. Briefly, the collected cells were resuspended in cold PBS/phosphatase inhibitors, followed by separation of the cytoplasmic fraction and the nuclear fraction, respectively. The separation of nuclear proteins was confirmed by the low expression of cytoplasmic protein $\alpha$-tubulin (Proteintech, 11224-1-AP) and the high expression of nuclear protein Lamin B1 (Proteintech, 12987-1-AP). The nuclear proteins extract was diluted in lysis buffer, then incubated with antibody overnight and the immune complexes precipitated with protein $\mathrm{A} / \mathrm{G}$ agarose for $3 \mathrm{~h}$ at $4{ }^{\circ} \mathrm{C}$. Immunoprecipitated proteins were analyzed by Western blotting.

\section{Cell viability and chemo-resistance assays}

Cells infected with LV-shAspp2 and LV-shNon were seeded into 96-well plates (5000 cells/well).Then cells were treated with different concentration 5-FU and $10 \mu \mathrm{M}$ Simvastatin or DMSO for $48 \mathrm{~h}$. Cell viability was measured by MTS assay reagent (CellTiter 96 AQueous One Solution Cell Proliferation Assay; Promega, Madison, WI, USA). The experiments have been repeated at least three times.

\section{Patient samples}

We performed a tissue microarray constructed by Shanghai Weiao Biotechnology Co., Ltd (Weiao Biotechnology Co, Shanghai, China). Eighty primary HCC samples made for microarray were obtained from patients who had undergone curative hepatic resection between 2008 and 2015. The clinicopathologic features of the patients were summarized in Supplementary Table 1. Other details about patient samples can be found in the supplementary material.

\section{Immunohistochemical staining}

The expressions of ASPP2, HMGCR, and HMGCS1 were analyzed with ImageScope system in formalin-fixed, paraffin-embedded sections of primary tumors. Briefly, the slides were dewaxed, hydrated,quenched endogenous peroxidase activity, retrieved antigen, blocked and incubated with the antibody against ASPP2 (1:50, A4480, Sigma-Aldrich), HMGCR (1:50, H300 sc-33827, Santa Cruz), or HMGCS1 (1:50, H-70 sc-33829, Santa Cruz) overnight at $4{ }^{\circ} \mathrm{C}$. Then, sections were rinsed and incubated with the working solution of horseradish peroxidase-labeled goat anti-rabbit for $1 \mathrm{~h}$ at $37^{\circ} \mathrm{C}$. After rinse for three times, diaminobenzidine colorimetric reagent solution from Dako (Carpinteria, CA) was used. Subsequently the slides were counterstained by hematoxylin and dehydrated in graded alcohol and mounted. The expression of ASPP2 and HMGCR were scored according to the signal intensity and distribution. Evaluation of immunostaining was independently performed by two experienced pathologists.

\section{Statistical analysis}

We use SPSS16.0 to analyze all data. The $\chi^{2}$ test was applied to assess qualitative variables 2-tailed Student's $t$ test and Wilcoxon rank sum test were used to valuate quantitative variables. Spearman rank test was used to determine correlations of two variables. Kaplan-Meier analysis was used to achieve Survival analyses of investigated patients. Difference in survival between groups was evaluated by the log-rank test. Univariate and multivariate analyses were based on the Cox proportional hazards regression model. All results were showed as the mean \pm SEM. All statistical tests were two-sided, and $P<0.05$ was considered statistically significant.

Other Material and Methods are available in the supplementary material and Methods.

\section{Acknowledgements \\ This work is supported by grants from National Nature Science Foundation of China $(81670573,81972252,81903065,81830052,81530053,81472719$, 81402030). Construction Project of Shanghai Key Laboratory of Molecular Imaging (18DZ2260400). Shanghai Municipal Education Commission (Class II Plateau Disciplinary Construction Program for Medical Technology of SUMHS, 2018-2020).}

\section{Author details \\ 'Shanghai Key Laboratory of Molecular Imaging, Shanghai University of Medicine and Health Sciences, 201318 Shanghai, China. ${ }^{2}$ Angecon Biotechnology Limited, 201318 Shanghai, China. ${ }^{3}$ Transplantation Center, Changzheng Hospital, Second Military Medical University, 200011 Shanghai, China. ${ }^{4}$ The Air Force Hospital from Northern Theater of PLA, Shenyang 110041, China. ${ }^{5}$ The Arctic Temple Clinic, Beijing Fourth Service Center, 8 Garden East Road, 100191 Beijing, China. ${ }^{6}$ Changhai Hospital, Second Military Medical University, 200438 Shanghai, China}

\section{Conflict of interest}

The authors declare that they have no conflict of interest. 


\section{Publisher's note}

Springer Nature remains neutral with regard to jurisdictional claims in published maps and institutional affiliations.

Supplementary Information accompanies this paper at (https://doi.org/ 10.1038/s41419-019-2054-7).

Received: 2 June 2019 Revised: 18 September 2019 Accepted: 1 October 2019

Published online: 04 November 2019

\section{References}

1. Hanahan, D. \& Weinberg, R. A. Hallmarks of cancer: the next generation. Cell 144, 646-674 (2011).

2. Simons, K. \& Ikonen, E. How cells handle cholesterol. Science 290, 1721-1726 (2000).

3. Beloribi-Djefaflia, S., Vasseur, S. \& Guillaumond, F. Lipid metabolic reprogramming in cancer cells. Oncogenesis 5, e189 (2016).

4. Kuzu, O. F., Noory, M. A. \& Robertson, G. P. The role of cholesterol in cancer. Cancer Res. 76, 2063-2070 (2016).

5. Murtola, T. J. et al. The importance of $L D L$ and cholesterol metabolism for prostate epithelial cell growth. PloS ONE 7, e39445 (2012).

6. Mo, H. \& Elson, C. E. Studies of the isoprenoid-mediated inhibition of mevalonate synthesis applied to cancer chemotherapy and chemoprevention. Exp. Biol. Med. 229, 567-585 (2004).

7. Cao, Z. et al. MYC phosphorylation, activation, and tumorigenic potential in hepatocellular carcinoma are regulated by HMG-CoA reductase. Cancer Res. 71, 2286-2297 (2011).

8. Moon, S.-H. et al. p53 represses the mevalonate pathway to mediate tumor suppression. Cell 176, 564-580. e519 (2019).

9. Mullen, P. J., Yu, R., Longo, J., Archer, M. C. \& Penn, L. Z. The interplay between cell signalling and the mevalonate pathway in cancer. Nat. Rev. Cancer 16, 718 (2016).

10. Ribas, V., García-Ruiz, C. \& Fernández-Checa, J. C. Mitochondria, cholesterol and cancer cell metabolism. Clin. Transl. Med. 5, 22 (2016).

11. Shimano, H. Sterol regulatory element-binding proteins (SREBPs): transcriptional regulators of lipid synthetic genes. Prog. Lipid Res. 40, 439-452 (2001).

12. Brown, M. S. \& Goldstein, J. L. The SREBP pathway: regulation of cholesterol metabolism by proteolysis of a membrane-bound transcription factor. Cell $\mathbf{8 9}$, 331-340 (1997).

13. Amemiya-Kudo, M. et al. Transcriptional activities of nuclear SREBP-1a,-1C, and2 to different target promoters of lipogenic and cholesterogenic genes. J. Lipid Res. 43, 1220-1235 (2002).

14. Yang, T. et al. Crucial step in cholesterol homeostasis: sterols promote binding of SCAP to INSIG-1, a membrane protein that facilitates retention of SREBPs in ER. Cell 110, 489-500 (2002).

15. Vives, $\mathrm{V}$. et al. ASPP2 is a haploinsufficient tumor suppressor that cooperates with p53 to suppress tumor growth. Genes Dev. 20, 1262-1267 (2006).

16. Samuels-Lev, Y. et al. ASPP proteins specifically stimulate the apoptotic function of p53. Mol. Cell 8, 781-794 (2001).

17. Sullivan, A. \& Lu, X. ASPP: a new family of oncogenes and tumour suppressor genes. Br. J. Cancer 96, 196 (2007).

18. Zhao, J. et al. Epigenetic silence of ankyrin-repeat-containing, SH3domain-containing, and proline-rich-region-containing protein 1 (ASPP1) and ASPP2 genes promotes tumor growth in hepatitis B virus-positive hepatocellular carcinoma. Hepatology 51, 142-153 (2010).

19. Wang, Y. et al. Autophagic activity dictates the cellular response to oncogenic RAS. Proc. Natl Acad. Sci. 109, 13325-13330 (2012).
20. Wang, Y. et al. ASPP2 controls epithelial plasticity and inhibits metastasis through $\beta$-catenin-dependent regulation of ZEB1. Nat. Cell Biol. 16, 1092 (2014).

21. Chen, R. et al. Downregulation of ASPP2 improves hepatocellular carcinoma cells survival via promoting BECN1-dependent autophagy initiation. Cell Death Dis. 7, e2512 (2016).

22. Sharpe, L. J. \& Brown, A. J. Controlling cholesterol synthesis beyond 3-hydroxy-3methylglutaryl-CoA reductase (HMGCR). J. Biol. Chem. R113, 479808 (2013). jbc.

23. Chung, J. Y. et al. Effect of HMGCR variant alleles on low-density lipoprotein cholesterol-lowering response to atorvastatin in healthy Korean Subjects. J. Clin. Pharmacol. 52, 339-346 (2012).

24. Ginestier, C. et al. Mevalonate metabolism regulates Basal breast cancer stem cells and is a potential therapeutic target. Stem Cells 30, 1327-1337 (2012).

25. Liu, M. et al. Transcriptional profiling reveals a common metabolic program in high-risk human neuroblastoma and mouse neuroblastoma sphere-forming cells. Cell Rep. 17, 609-623 (2016).

26. Wang, $X$. et al. MYC-regulated mevalonate metabolism maintains brain tumor-initiating cells. Cancer Res. 77, 4947-4960 (2017).

27. $\mathrm{Xu}$, L. et al. ASPP2 suppresses stem cell-like characteristics and chemoresistance by inhibiting the Src/FAK/Snail axis in hepatocellular carcinoma. Tumor Biol. 37, 13669-13677 (2016).

28. Sassano, A. \& Platanias, L. C. Statins in tumor suppression. Cancer Lett. 260 $11-19$ (2008).

29. Chan, K. K., Oza, A. M. \& Siu, L. L. The statins as anticancer agents. Clin. Cancer Res. 9, 10-19 (2003).

30. Terris, B., Cavard, C. \& Perret, C. EpCAM, a new marker for cancer stem cells in hepatocellular carcinoma. J. Hepatol. 52, 280-281 (2010).

31. Yamashita, T. et al. EpCAM-positive hepatocellular carcinoma cells are tumorinitiating cells with stem/progenitor cell features. Gastroenterology 136, 1012-1024. e1014 (2009).

32. Horton, J. D., Goldstein, J. L. \& Brown, M. S. SREBPs: activators of the complete program of cholesterol and fatty acid synthesis in the liver. J. Clin. Investig. 109, 1125-1131 (2002)

33. Vousden, K. H. \& Ryan, K. M. p53 and metabolism. Nat. Rev. Cancer 9, 691 (2009).

34. Freed-Pastor, W. A. et al. Mutant p53 disrupts mammary tissue architecture via the mevalonate pathway. Cell 148, 244-258 (2012).

35. Bergamaschi, D. et al. ASPP1 and ASPP2: common activators of p53 family members. Mol. Cell. Biol. 24, 1341-1350 (2004).

36. Sottocornola, R. et al. ASPP2 binds Par-3 and controls the polarity and proliferation of neural progenitors during CNS development. Dev. Cell 19, 126-137 (2010).

37. Cong, W. et al. ASPP2 regulates epithelial cell polarity through the PAR complex. Curr. Biol. 20, 1408-1414 (2010).

38. Wang, Y. et al. ASPP1 and ASPP2 bind active RAS, potentiate RAS signalling and enhance p53 activity in cancer cells. Cell Death Differ. 20, 525 (2013).

39. Wang, Z. et al. N terminus of ASPP2 binds to Ras and enhances Ras/Raf/MEK ERK activation to promote oncogene-induced senescence. Proc. Natl Acad. Sci. 110, 312-317 (2013).

40. Trigiante, G. \& Lu, X. ASPPs and cancer. Nat. Rev. Cancer 6, 217 (2006).

41. Godin-Heymann, N., Wang, Y., Slee, E. \& Lu, X. Phosphorylation of ASPP2 by RAS/MAPK pathway is critical for its full pro-apoptotic function. PLOS ONE 8 , e82022 (2013).

42. Turnquist, C. et al. STAT1-induced ASPP2 transcription identifies a link between neuroinflammation, cell polarity, and tumor suppression. Proc. Natl Acad. Sci. 111, 9834-9839 (2014).

43. Lu, M. et al. A code for RanGDP binding in ankyrin repeats defines a nuclear import pathway. Cell 157, 1130-1145 (2014).

44. Shamma, A. et al. Rb regulates DNA damage response and cellular senescence through E2F-dependent suppression of $\mathrm{N}$-ras isoprenylation. Cancer Cell 15, 255-269 (2009). 